\title{
Roles of VE-Cadherin in Hypoxia Induced Injury of Pulmonary Microvascular Endothelial Barrier
}

\author{
Lizhe Zhong, PhD, ${ }^{1}$ Xiurong Gao, $\mathrm{PhD},{ }^{2}$ Yongli Chen, $\mathrm{PhD},{ }^{1}$ Zhaoxiang Yu, $\mathrm{PhD},{ }^{3}$ Shuo Jin, $\mathrm{PhD},{ }^{4}$ \\ Chunlei Zhu, $\mathrm{PhD}^{4}$ \\ ${ }^{1}$ Department of Thoracic Surgery, Affiliated Hospital of Beihua University, Jilin 132013, China; \\ ${ }^{2}$ Department of Second Cardiovascular, Qianguo County Hospital, Songyuan 138000, China; \\ ${ }^{3}$ Department of Anesthesiology, Affiliated Hospital of Beihua University, Jilin 132013, China; \\ ${ }^{4}$ Department of Hand and Foot Surgery, Affiliated Hospital of Beihua University, Jilin 132013, China
}

\section{ABSTRACT}

Background: Hypoxia induced injury of pulmonary microvascular endothelial barrier is closely related to the pathogenesis of acute lung injury after lung transplantation. VE-cadherin is an important structural molecule for pulmonary microvascular endothelial barrier. In this study, we aim to investigate the roles of $\mathrm{VE}$-cadherin in hypoxia induced injury of pulmonary microvascular endothelial barrier.

Methods: Rat model of hypoxia and cultured pulmonary microvascular endothelial cells (PMVECs) were utilized. Determination of PMVECs apoptosis, skeleton combination was conducted to verify the effects of hypoxia on injury of pulmonary microvascular endothelial barrier. In addition, VE-cadherin expression was modulated by administration of siRNA in order to investigate the roles of VE-cadherin in hypoxia induced PMVECs apoptosis and skeleton recombination.

Results: Our data indicated that expression of VE-cadherin was down-regulated in hypoxia-exposed PMVECs. Whereas, in the cells treated using siRNA, down-regulation of VE-cadherin did not trigger PMVECs apoptosis, but it increased the sensitivity of PMVECs to the hypoxia induced apoptosis. In cases of hypoxia, the expression of VE-cadherin was significantly down-regulated, together with endothelial skeleton recombination and increase of permeability, which then triggered endothelial barrier dysfunction.

Conclusions: These data verify that VE-cadherin expression played an important role in hypoxia induced PMVECs apoptosis and cellular skeletal recombination.
Received October 19, 2020; accepted November 18, 2020.

Correspondence: Chunlei Zbu, No. 12, Fiefangzhong Road, Department of Hand and Foot Surgery, Affiliated Hospital of Beihua University, Chuanying District, Filin 132013, China, Telephone +86-432-62166000 (e-mail: luckdbzcl@sina.com).

\section{INTRODUCTION}

Most of the studies on lung transplantation have been focused on the immunological rejection. Little is known about the molecular mechanism and prevention of vascular endothelial injury after pulmonary ischemia and hypoxia. To date, no effective treatment options and prevention methods are available for the ischemia-reperfusion injury of lung.

Hypoxia induced injury of pulmonary microvascular endothelial cells (PMVECs), such as cellular apoptosis and barrier function injury, which then triggered infiltration of inflammatory cells and release of inflammatory factors that were considered as the major causes for acute lung injury after pulmonary transplantation. PMVECs, localized in the internal side of the blood air barrier [Stevens 2011], formed the endothelial barrier together with the adjacent endothelial cells. The adherens junction formed by adjacent PMVECs was the most complex structure in the endothelial junction, with its function serving as an important type of junction [Taveau 2008].

VE-cadherin is the major structural molecule for the adherens junction of vascular endothelial cells [Abu Taha 2014]. Its expression could trigger injury of vascular endothelial barrier function and increase of microvascular permeability, which played important roles in the pathogenesis of various diseases [Kandasamy 2015; Huang 2015]. In this study, RNAi technique was utilized to modulate the expression of VE-cadherin, and then the roles of VE-cadherin in hypoxia induced PMVECs apoptosis and skeletal recombination was investigated. Our findings indicated that hypoxia induced down-regulation of VE-cadherin and increase of sensitivity of PMVECs to the hypoxia induced apoptosis. Besides, VE-cadherin down-regulation resulted in recombination of PMVECs skeleton. These indicated that VE-cadherin played crucial roles in hypoxia induced endothelial barrier dysfunction.

\section{MATERIALS AND METHODS}

Establishing hypoxia induced pulmonary injury model in rats: Male Sprague-Dawley rats (7 weeks old) were anesthetized after trachea cannula connecting the respirator 
with a respiratory frequency of $80-90 / \mathrm{min}$. The respiratory exchange ratio was set at 1:2-1:3 with a working pressure of 2-4 kPA as previously described [Wu 2016].

Rats were divided into four groups: (i) sham control group $(N=7)$, which were subjected to sham operation; (ii) ischemia for $30 \mathrm{~min}(N=7)$; (iii) ischemia for 3 h group ( $N$ $=7$ ); and (iv) ischemia for $12 \mathrm{~h}$ group $(N=7)$. The chest was entered by the 4th intercostal space at the left body side, followed by heparinization and ligation of left inferior lung lobe for $30 \mathrm{~min}, 3 \mathrm{~h}$, and $12 \mathrm{~h}$, respectively. The animals were sacrificed at these time points to obtain the samples from left inferior lung and serum samples. The animal study protocols were approved by the Ethical Committee of the Beihua University Hospital and all animal handling was performed in accordance with the Jilin Directive for Animal Research and the current Guidelines for the Care and Use of Laboratory Animals published by the National Institutes of Health. Rats were sacrificed by decapitation after being successfully anesthetized with $2 \%$ Phenobarbital $(40 \mathrm{mg} / \mathrm{Kg})$. Unnecessary suffering was avoided as far as possible.

Cell culture: Rat PMVECs purchased from Bena Culture Collection (category No. BNCC338210, Suzhou, China) were cultured using the standard method as previously described [Wu 2016]. Briefly, cells were cultured in endothelial culture medium (No. 1001, Sciencell) supplemented with $5 \%$ fetal bovine serum (FBS, No. 0025, Sciencell), $1 \%$ endothelial cell growth supplement (No. 1052, Sciencell) and $1 \%$ penicillin/streptomycin solution (No. 0503, Sciencell) in $21 \%$ oxygen and $5 \%$ carbon dioxide at $37^{\circ} \mathrm{C}$. PMVECs (P2-4) were expanded in monolayers in cell culture bottle. The culture medium was changed to a serum-free solution for $24 \mathrm{~h}$ prior to usage.

To investigate the roles of $\mathrm{VE}$-cadherin in hypoxia induced PMVECs apoptosis in vitro, cells were divided into four groups, including the control group; hypoxia interference group treated by $5 \% \mathrm{O}_{2}$; VE-cadherin knockdown group subject to knockdown of VE-cadherin by RNAi technique; and hypoxia interference $+\mathrm{VE}$-cadherin knockdown group subject to $5 \% \mathrm{O}$, and knockdown of VE-cadherin by RNAi.

Knockdown of VE-cadherin expression by RNAi: Smart Pool siRNA for VE-cadherin was obtained from Dharmacon (Lafayette, CO). Transient transfection of PMVECs with sequence specific siRNA or scrambled control was completed using oligofectamine (Invitrogen, Carlsbad, CA), $200 \mathrm{~nm}$ siRNA and $1.5 \times 10^{6}$ cells per reaction as previously described [O'Leary 2010]. Cells were then treated with DNR, and collected at $72 \mathrm{~h}$ for evaluation of apoptosis and cytoskeleton.

Histopathological examination: After lung tissues sample collection, HE staining was performed to determine the pulmonary injury extent. The images were obtained from a BX51 light microscope (Olympus Corporation, Tokyo, Japan). Meanwhile, an Eclipse 80i fluorescence microscope (Nikon, Tokyo, Japan) was utilized for the imaging collection and analysis.

Western blotting analysis: VE-cadherin was detected using a standard Western blot protocol to determine the expression of VE-cadherin protein [Orsenigo 2012]. The transferred membrane was blocked with $10 \%$ skimmed milk for $1 \mathrm{~h}$ at room temperature, and then was incubated with the primary antibody against VE-cadherin $(1: 1000)$ and $\beta$-actin $(1: 1000)$ overnight at $4^{\circ} \mathrm{C}$. After incubation with the horseradish peroxidase-conjugated secondary antibody (1:5000) for $1 \mathrm{~h}$ at room temperature, the immunoblotting signals were visualized using a Luminescent Detection kit (Vigorous Biotechnology, Beijing, China).

TUNEL assay: Cellular apoptosis was determined using TUNEL assay as previously described [Wu 2018; Takeda 2015]. Sections were incubated with TUNEL reaction mixture (11684817910, Roche, Germany), according to the manufacturer's instructions. Sections were then observed using an Eclipse 80i fluorescence microscope (Nikon, Tokyo, Japan). Subsequently, 10 visual fields randomly were selected and observed under a magnification of 200x.

Immunofluorometric assay: Immunofluorometric assay was carried out to determine the cellular skeleton. The coverslip wrapped by the PMVECs was fixed using paraformaldehyde, and then Actin-Tracker Green (1:100, C10330, Beyotime) was added and incubated at room temperature for 30 min in the dark. After washing with PBS containing $0.1 \%$ Triton X-100, the cells were counterstained. The mixture was then incubated at room temperature for $30 \mathrm{~min}$. Fluoromount-G (category No. 0100-01, Birmingham, USA) was used to block the coverslip [Audia 2013]. Finally, the images were observed using a BX53 fluorescence microscope (Olympus, Tokyo, Japan).

Determination of pulmonary microvascular permeability: Pulmonary microvascular permeability was evaluated through administration of azovan coerulen via caudal vein injection. The content of azovan coerulen was determined under a wavelength of $625 \mathrm{~nm}$ utilized to evaluate the extent of pulmonary microvascular permeability.

Measurement of laboratory indices: Indices including expression of malondialdehyde (MDA), superoxide dismutase (SOD) activity, and the weight-to-dry weight (W/D) ratio in pulmonary tissues were detected. The mean value was obtained after at least three triplicates.

Statistical analysis: SPSS 20.0 software was utilized for the statistical analysis. Quantitative data was presented as the mean \pm standard error of mean (SEM). Statistically significant differences in mean values were tested by Student's t-test or by one-way ANOVA using Dunnett's test in multiple comparisons. $P<0.05$ was considered to be statistically significant.

\section{RESULTS}

Hypoxia induced pulmonary injury and increase of pulmonary microvascular permeability: With the extension of ischemic duration, the pulmonary microvascular permeability in lung tissues showed significant increase combined with obvious pulmonary edema. HE staining indicated that there was obvious pulmonary interstitial edema in the model group. In addition, there was massive infiltration of cells in the lung tissues in the hypoxia group. (Figure 1) Moreover, remarkable elevation was noticed in the pulmonary microvascular permeability and W/D ratios in lung tissues. (Table 1) The MDA 
Table 1. Determination of pulmonary microvascular permeability and $W / D$ in each group

\begin{tabular}{lcc}
\hline Group & $\begin{array}{c}\text { Pulmonary microvascular } \\
\text { permeability }(\mu \mathrm{g} / \mathrm{mg})\end{array}$ & W/D \\
\hline Normal control & $0.049 \pm 0.003$ & $3.28 \pm 0.61$ \\
Hypoxia 30 min group & $0.050 \pm 0.006$ & $3.31 \pm 0.52$ \\
Hypoxia 3h group & $0.107 \pm 0.02 *$ & $4.93 \pm 0.70 *$ \\
Hypoxia 12h group & $0.171 \pm 0.04 *$ & $5.53 \pm 0.85^{*}$ \\
\hline
\end{tabular}

$* P<0.05$, compared with normal control

Table 2. Determination of MDA, and SOD in each group

\begin{tabular}{lcc}
\hline Group & MDA (nmol/mgprot) & SOD (U/mgprot) \\
\hline Normal control & $1.31 \pm 0.41$ & $51.49 \pm 7.53$ \\
Hypoxia 30 min group & $1.34 \pm 0.39$ & $49.77 \pm 8.25$ \\
Hypoxia 3h group & $2.94 \pm 0.58^{*}$ & $34.63 \pm 6.03^{*}$ \\
Hypoxia 12h group & $4.41 \pm 0.78^{*}$ & $28.35 \pm 7.11 *$ \\
\hline
\end{tabular}

$* P<0.05$, compared with normal control

showed significant elevation about 3 hrs after hypoxia, and SOD showed significant decrease. (Table 2) All these indicated the presence of obvious lung injury.

Effect of hypoxia on VE-cadherin expression in lung tissues and PMVECs: With the extension of hypoxia duration, VE-cadherin expression showed gradual decrease compared with the normal control (Figure 2A). (Figure 2) In this section, we investigated the expression of protein level in cultured PMVECs in order to validate the effects of hypoxia on VE-cadherin expression in PMVECs. Our data showed that hypoxia could down-regulate the VE-cadherin protein expression in PMVECs (Figure 2B).

Roles of VE-cadherin in PMVEC apoptosis induced by hypoxia: According to the previous description, hypoxia triggered apoptosis of PMVECs [Guo 2015]. As an important structural molecule in endothelial adherens junction, VEcadherin played important roles in apoptosis of endothelial cells. On this basis, we utilized RNAi to modulate the expression of VE-cadherin in order to evaluate its roles in hypoxia induced PMVEC apoptosis and permeability. As shown in Figure 3, inhibition of VE-cadherin could not trigger PMVEC apoptosis. (Figure 3) However, in the presence of hypoxia, it could enhance apoptosis of PMVECs. This indicated that inhibition VE-cadherin expression promoted the apoptosis of PMVECs.

Roles of VE-cadherin in PMVEC skeletal recombination induced by hypoxia: There was accumulation of F-actin fiber in PMVECs in normal rats in the peripheral tissues of adjacent cell membrane. (Figure 4) In addition, the microfibril in cytoplasm was arranged in a regular manner. In cases of hypoxia,

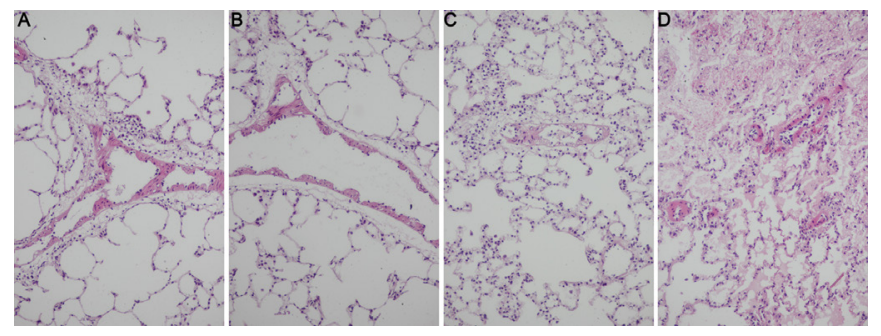

Figure 1. Hypoxia induced animal model based on ligation of left inferior lung lobe. Obvious edema and massive cellular infiltration was noticed in the lung tissues in the model group after HE staining. (A) sham control group; (B) ischemia for $30 \mathrm{~min}$; (C) ischemia for 3 h group; (D) ischemia for $12 \mathrm{~h}$ group

the expression of VE-cadherin was down-regulated, and obvious changes were noticed in the profile and distribution of F-actin fibers, which were featured by stress fiber, filipodium, and lamellipodia. There were massive stress fibers in the cytoplasm after knockdown of VE-cadherin, together with endothelial cell shrinkage and formation of intracellular fissures. On this basis, we speculated that the expression of VE-cadherin played crucial roles in the stability of PMVECs skeleton.

\section{DISCUSSION}

Hypoxia is a type of inevitable injury factor for organ transplantation. It affects the functional recovery of implanted organs and is closely related to acute rejection and chronic implanted organ hypofunction [Requiao-Moura 2011]. Recently, the standardization of donation after cardiac death (DCD) contributed to the organ transplantation, but there still are some challenges. This extends the organ source for the patients with end-stage diseases, however, hypoxia induced injury during storage, transmission and transplantation may hamper the efficiency of organ utilization [Elgharably 2015].

Hypoxia may trigger pulmonary microvascular endothelial barrier function, including endothelial apoptosis and increase of permeability, which then resulted in acute lung injury. Therefore, we aimed to investigate the correlation between hypoxia and pulmonary microvascular endothelial barrier injury. To our best knowledge, pulmonary microvascular endothelial barrier can limit the large molecules generated in endothelial cells and circulating cells in blood other than penetrating into the tissues. Meanwhile, the permeability of endothelial cells mainly was modulated by the endothelial adherens junction.

The endothelial adherens junction is the most important type of cellular junction. It is formed by VE-cadherin catenin complex consisting of VE-cadherin, catenin, and actin microfilament [Padmanabhan 2015]. VE-cadherin molecule consisted of 780 amino acids, which was divided into extracellular region, intracellular region, and transmembrane domain. Specifically, the amino terminal (extracellular region) showed isophil junction [Baumgartner 2000], which could form adherens junction with the VE-cadherin related molecules in the adjacent vascular endothelial cells. These demonstrated 
A

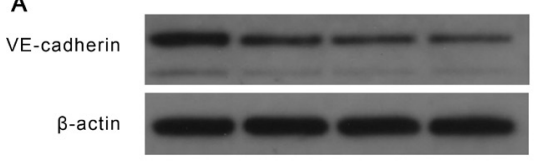

VE-cadherin/ß-actin

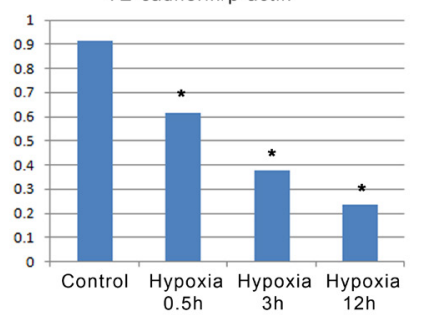

B VE-cadherin
$\beta$-actin
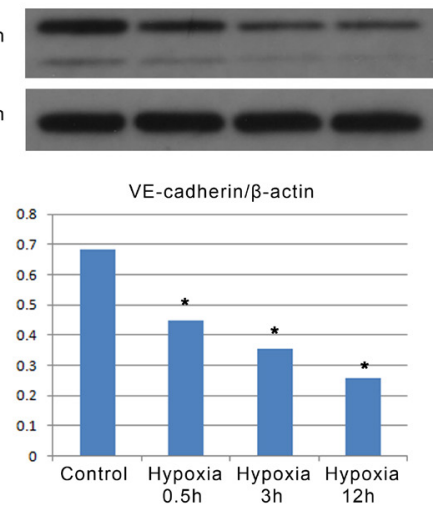

Figure 2. Hypoxia induced significant down-regulation of VE-cadherin in lung tissues and cultured PMVECs. (A) In the model group, the expression of VEcadherin in lung tissues showed significant decrease compared with control. (B) In the presence of hypoxia, the VE-cadherin expression in cultured PMVECs showed significant decrease. ${ }^{*} P<0.05$ versus control group

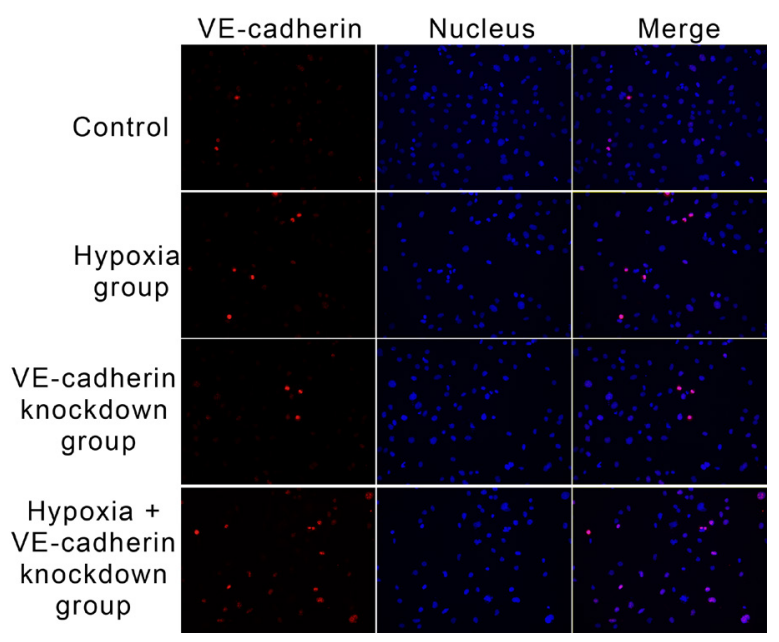

Figure 3. Down-regulation of VE-cadherin increased the sensitivity of PMVECs to the hypoxia-induced apoptosis. TUNEL revealed apoptosis of PMVECs. $* P<0.05$ versus control group; $\# P<0.05$ versus hypoxia group

that VE-cadherin contributed to intact of endothelial barrier. In recent studies, VE-cadherin could modulate the cellular viability and involve in signaling transmission [Birdsey 2008; Carmeliet 1999]. Therefore, it has been considered as an important target for endothelial barrier function. VE-cadherin expression alternation may be associated with biological behaviors of PMVECs and barrier function.

In a previous study, hypoxia could induce apoptosis of PMVECs [Guo 2015], whereas little is known about the VEcadherin serving an important molecule role in the adherens junction. In this study, the expression of VE-cadherin was down-regulated in the PMVECs under hypoxia conditions. Our study further indicated that after knockdown of VE-cadherin using RNAi, the PMVECs viability showed no statistical difference, but it could contribute to the hypoxia induced PMVECs apoptosis accordingly. These findings implied that VE-cadherin played protective roles on PMVECs, and its

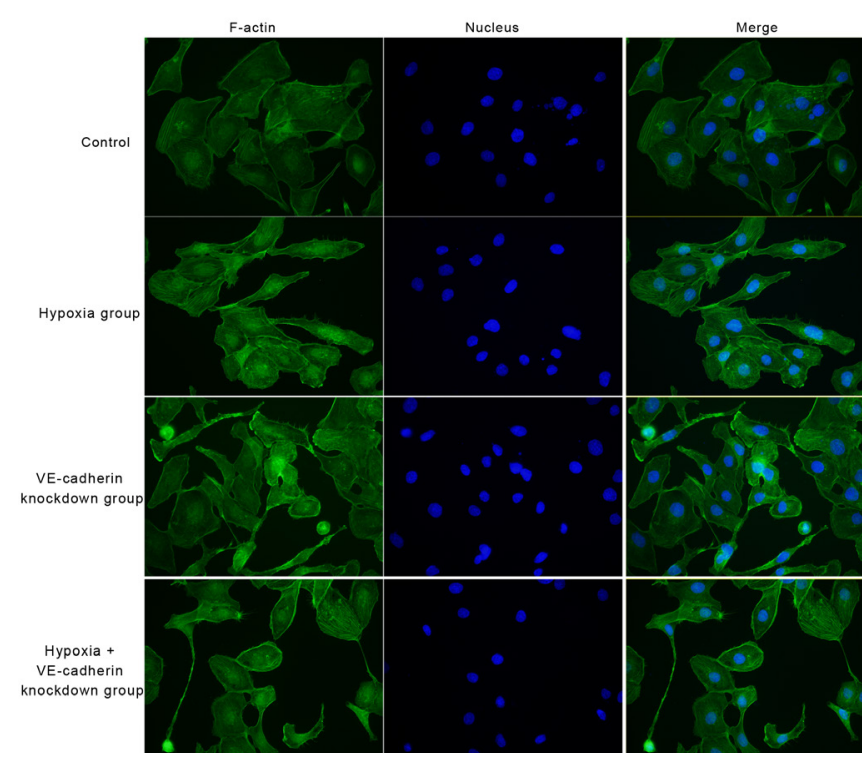

Figure 4. VE-cadherin down-regulation hampered the skeleton of PMVECs. In the presence of hypoxia, the VE-cadherin was down-regulated in PMVECs. Additionally, there were significant changes in the distribution and profile of F-actin in PMVECs, which were featured by presence of stress fiber, filipodium and lamellipodia. After VE-cadherin knockdown, massive stress fiber was noticed in PMVECs together with endothelial shrinkage and formation of inter-cellular fissure.

down-regulation could increase the sensitivity of endothelial cells to apoptosis.

Endothelial barrier intact has been reported to be associated with the endothelial skeleton [Kasa 2015], while VE-cadherin is crucial for the stability of cellular skeleton [Orsenigo 2012; Weidert 2014; Wallez 2007]. Our findings indicated that the F-actin in the PMVECs of normal rats was mainly accumulated in the peripheral tissues of the adjacent membrane, and the arrangement of the microfilament in the cytoplasm was regular. In cases of hypoxia, the expression of VEcadherin was down-regulated, and the profile and distribution 
of $\mathrm{F}$-actin showed obvious changes, which led to formation of stress fiber, filipodium and lamellipodia. Whereas, in the presence of VE-cadherin knockdown, massive stress fiber was noticed in the cytoplasm together with shrinkage of endothelial cells and formation of intracellular space. This indicated that stable expression of VE-cadherin was crucial for the stability of PMVEC skeleton.

There were some limitations to this study. First of all, the sample size was not large for the in vivo experiments. To solve this problem, in our subsequent study, we may expand the sample size. Secondly, we cannot identify the exact mechanism of VE-cadherin down-regulation in PMVECs. In the future, we will focus on it.

In summary, hypoxia could induce pulmonary injury through hampering the endothelial barrier function. For the mechanism, it may be related to the down-regulation of VE-cadherin in PMVECs in presence of hypoxia, which in turn contributed to the sensitivity of PMVECs to the hypoxia induced apoptosis. Besides, VE-cadherin down-regulation promoted skeletal recombination of PMVECs.

\section{ACKNOWLEDGEMENT}

This work was supported by grants from the Foundation of Jilin Provincial Department of Education, China (No. JJKH20170055K), the Foundation of Health Department of Jilin Province, China (No. 2017J080).

\section{REFERENCES}

Abu Taha A, Schnittler HJ. 2014. Dynamics between actin and the VEcadherin/catenin complex: novel aspects of the ARP2/3 complex in regulation of endothelial junctions. Cell adhesion \& migration. 8(2):125-35.

Audia JP, Lindsey AS, Housley NA, et al. 2013. In the absence of effector proteins, the Pseudomonas aeruginosa type three secretion system needle tip complex contributes to lung injury and systemic inflammatory responses. PloS one. 8(11):e81792.

Baumgartner W, Hinterdorfer P, Ness W, et al. 2000. Cadherin interaction probed by atomic force microscopy. Proceedings of the National Academy of Sciences of the United States of America. Apr 11. 97(8):4005-10.

Birdsey GM, Dryden NH, Amsellem V, et al. 2008. Transcription factor Erg regulates angiogenesis and endothelial apoptosis through VE-cadherin. Blood. Apr 1. 111(7):3498-506.

Carmeliet P, Lampugnani MG, Moons L, et al. 1999. Targeted deficiency or cytosolic truncation of the VE-cadherin gene in mice impairs VEGFmediated endothelial survival and angiogenesis. Cell. Jul 23. 98(2):147-57.

Elgharably H, Shafii AE, Mason DP. 2015. Expanding the donor pool: donation after cardiac death. Thoracic surgery clinics. 25(1):35-46.

Guo H, Cao J, Li J, et al. 2015. Lymphocytes from intermittent hypoxiaexposed rats increase the apoptotic signals in endothelial cells via oxidative and inflammatory injury in vitro. Sleep \& breathing $=$ Schlaf \& Atmung. Sep. 19(3):969-76.

Huang Y, Tan Q, Chen R, Cao B, Li W. 2015. Sevoflurane prevents lipopolysaccharide-induced barrier dysfunction in human lung microvascular endothelial cells: Rho-mediated alterations of VE-cadherin. Biochemical and biophysical research communications. Dec 4-11. 468(1-2):119-24.

Kandasamy K, Escue R, Manna J, Adebiyi A, Parthasarathi K. 2015. Changes in endothelial connexin 43 expression inversely correlate with microvessel permeability and VE-cadherin expression in endotoxin-challenged lungs. American journal of physiology Lung cellular and molecular physiology. Sep 15. 309(6):L584-92.

Kasa A, Csortos C, Verin AD. 2015. Cytoskeletal mechanisms regulating vascular endothelial barrier function in response to acute lung injury. Tissue barriers. 3(1-2):e974448.

O'Leary H, Akers SM, Piktel D, et al. 2010. VE-cadherin Regulates Philadelphia Chromosome Positive Acute Lymphoblastic Leukemia Sensitivity to Apoptosis. Cancer microenvironment: official journal of the International Cancer Microenvironment Society. Mar 2. 3(1):67-81.

Orsenigo F, Giampietro C, Ferrari A, et al. 2012. Phosphorylation of VE-cadherin is modulated by haemodynamic forces and contributes to the regulation of vascular permeability in vivo. Nature communications. 3:1208.

Padmanabhan A, Rao MV, Wu Y, Zaidel-Bar R. 2015. Jack of all trades: functional modularity in the adherens junction. Current opinion in cell biology. Oct. 36:32-40.

Requiao-Moura LR, Durao Mde S, Tonato EJ, et al. 2011. Effects of ischemia and reperfusion injury on long-term graft function. Transplantation proceedings. Jan-Feb. 43(1):70-3

Stevens T. 2011. Functional and molecular heterogeneity of pulmonary endothelial cells. Proceedings of the American Thoracic Society. Nov $8(6): 453-7$.

Takeda K, Uchiyama K, Kinukawa M, Tagami T, Kaneda M, Watanabe S. 2015. Evaluation of sperm DNA damage in bulls by TUNEL assay as a parameter of semen quality. The Journal of reproduction and development. 61(3):185-90.

Taveau JC, Dubois M, Le Bihan O, et al. 2008. Structure of artificial and natural VE-cadherin-based adherens junctions. Biochemical Society transactions. Apr. 36(Pt 2):189-93.

Wallez Y, Cand F, Cruzalegui F, et al. 2007. Src kinase phosphorylates vascular endothelial-cadherin in response to vascular endothelial growth factor: identification of tyrosine 685 as the unique target site. Oncogene. Feb 15. 26(7):1067-77.

Weidert E, Pohler SE, Gomez EW, Dong C. 2014. Actinomyosin contraction, phosphorylation of $\mathrm{VE}$-cadherin, and actin remodeling enable melanoma-induced endothelial cell-cell junction disassembly. PloS one. 9(9):e108092.

Wu XM, Ji KQ, Wang HY, et al. 2018. MicroRNA-339-3p alleviates inflammation and edema and suppresses pulmonary microvascular endothelial cell apoptosis in mice with severe acute pancreatitis-associated acute lung injury by regulating Anxa3 via the Akt/mTOR signaling pathway. Journal of cellular biochemistry. Aug. 119(8):6704-6714.

Wu Z, Liu H, Ren W, Dai F, Chang J, Li B. 2016. VE-cadherin involved in the pulmonary microvascular endothelial cell barrier injury induced by angiotensin II through modulating the cellular apoptosis and skeletal rearrangement. American journal of translational research. 8(10):4310-4319.

Wu ZY, Yao Y, Hu R, Dai FF, Zhang H, Mao ZF. 2016. Cyclic adenosine monophosphate-protein kinase A signal pathway may be involved in pulmonary aquaporin-5 expression in ischemia/reperfusion rats following deep hypothermia cardiac arrest. Genetics and molecular research: GMR. Feb 5. 15(1). 